\title{
Some properties of Fuzzy Derivative (I)
}

\author{
Salah Mahdi Ali \\ Department of Statistics and Information College of Computer Science and Mathematics University of Al- \\ Qadisiya, Diwaniya, Iraq.
}

Abstract: In [3], the fuzzy derivative was defined by using Caratheodory's derivative notion and a few basic properties of fuzzy derivative was proved. In this paper, we will a completion to prove for some properties of the subject and discussion Rolle's theorem and Generalized Mean-Value Theorem in fuzzy derivative and we given some applications of the Mean Value Theorem.

Keywords: Chain Rule, Critical point theorem, Rolle's Theorem, Generalized Mean-Value Theorem. MSC (2010): 54A40,46S40

\section{Preliminaries}

Definition 1.1.[5] Let $\boldsymbol{X}$ be a vector space over the field $\boldsymbol{F}$ of real or complex numbers, $(\boldsymbol{X}, \boldsymbol{T})$ be a fuzzy topological space, if the two mappings $X \times X \rightarrow X,(x, y) \mapsto x+y$ and $X \times F \rightarrow X,(\alpha, x) \mapsto \alpha x$ are fuzzy continuous, where $\boldsymbol{F}$ is the induced fuzzy topology of the usual norm, then $(\boldsymbol{X}, \boldsymbol{T})$ is said to be a fuzzy topological vector space over the field $\boldsymbol{F}$.

Definition 1.2. (Caratheodory). Let $f:(a, b) \subseteq R \rightarrow R$ be a function and $\boldsymbol{c} \in(\boldsymbol{a}, \boldsymbol{b})$, then $f$ is said to be differentiable at a point $\boldsymbol{c}$ if there exist a function $\boldsymbol{U}_{\boldsymbol{c}}$ that is continuous at $\boldsymbol{x}=\boldsymbol{c}$ and satisfies the relation $f(x)-f(c)=U_{c}(x)(x-c)$ for all $x \in(a, b)$.

We will usually write $\boldsymbol{U}(\boldsymbol{x})$ instead of $\boldsymbol{U}_{\boldsymbol{c}}(\boldsymbol{x})$, since there to be little chance of confusion, but we must remember that the function $\boldsymbol{U}$ depend on the point $\boldsymbol{c}$.

Definition 1.3. Let $R$ be the field of real numbers and $(\boldsymbol{R}, \boldsymbol{T})$ be a fuzzy topological vector space over the field $\boldsymbol{R}$. A function $\boldsymbol{f}: \boldsymbol{R} \rightarrow \boldsymbol{R}$ is said to be fuzzy differentiable at a point $\boldsymbol{c}$ if there is a function $\boldsymbol{U}$ that is fuzzy continuous at $x=c$ and have $f(x)-f(c)=U(x)(x-c)$ for all $x \in R$.

$\boldsymbol{U}(\boldsymbol{c})$ is said to be the fuzzy derivative of $\boldsymbol{f}$ at $\boldsymbol{c}$ and denoted $\boldsymbol{f}^{\prime}(\boldsymbol{c})=\boldsymbol{U}(\boldsymbol{c})$.

\section{Main Results}

Theorem 2.1. If $\boldsymbol{f}$ is fuzzy differentiable at a point $\boldsymbol{c}$, then $\boldsymbol{f}$ is fuzzy continuous at a point $\boldsymbol{c}$.

Proof. Assume $f$ is fuzzy differentiable at a point $\boldsymbol{c}$, then there is a fuzzy continuous function, say $\boldsymbol{\varphi}$ and satisfies the relation

$$
f(x)-f(c)=\varphi(x)(x-c) \text { for all } x \in R
$$

Since $\varphi$ is fuzzy continuous at $c$, then $\varphi(x)$ is nearly equal to $\varphi(c)=f^{\prime}(c)$ if $x$ is near $c$.

Replacing $\varphi(x)$ by $f^{\prime}(\boldsymbol{c})$ in (1), we obtain the equation $f(x)=f(c)+f^{\prime}(c)(x-c)$

Which should be approximately correct when $(\boldsymbol{x}-\boldsymbol{c})$ is small ( i.e. If $\boldsymbol{f}$ is differentiable at $\boldsymbol{c}$, then $\boldsymbol{f}$ is approximately a linear function near $\boldsymbol{c}$. 
Theorem 2.2. (Chain Rule). [3] If $\boldsymbol{f}$ is fuzzy differentiable at a point $\boldsymbol{c}$ and $\boldsymbol{g}$ is fuzzy differentiable at a point $f(c)$, then $\boldsymbol{h}=\boldsymbol{g} \circ f$ is also fuzzy differentiable at a point $\boldsymbol{c}$ and $\boldsymbol{h}^{\prime}(\boldsymbol{c})=g^{\prime}(f(c)) \boldsymbol{f}^{\prime}(\boldsymbol{c})$.

Theorem 2.3. If $\boldsymbol{f}$ and $\boldsymbol{g}$ are fuzzy differentiable at a point $\boldsymbol{c}$, then

(1) $(f \pm g)^{\prime}(c)=f^{\prime}(c) \pm g^{\prime}(c)$,

(2) $(f g)^{\prime}(c)=f(c) g^{\prime}(c)+g(c) f^{\prime}(c)$,

(3) $\left(\frac{f}{g}\right)^{\prime}(c)=\frac{f^{\prime}(c) g(c)-g^{\prime}(c) f(c)}{g^{2}(c)}, g(c) \neq 0$

Proof. We shall prove (2)

By using (Definition 3) there are two function $\varphi$ and $\psi$ both are fuzzy continuous at a point $\boldsymbol{c}$ and

$$
\begin{aligned}
& f(x)=f(c)+\varphi(x)(x-c) \\
& g(x)=g(c)+\psi(x)(x-c)
\end{aligned} \text { for all } x \in(a, b) .
$$

Now,

$$
\begin{aligned}
& f(x) g(x)=[f(c)+\varphi(x)(x-c)][g(c)+\psi(x)(x-c)] \\
& =f(c) g(c)+[f(c) \psi(x)+g(c) \varphi(x)+\varphi(x) \psi(x)(x-c)](x-c)
\end{aligned}
$$

Then $(f g)(x)=(f g)(c)+\eta(x)(x-c)$

Where $\eta(x)=f(c) \psi(x)+g(c) \varphi(x)+\varphi(x) \psi(x)(x-c)$, which is fuzzy continuous at $c$. If $x$ is near $c$, then $\varphi(x)$ is nearly equal to $\varphi(c)=f^{\prime}(c)$ and $\psi(x)$ is nearly equal to $\psi(c)=g^{\prime}(c)$, finally $(f g)^{\prime}(x)=\left.\eta(x)\right|_{x=c}=f(c) g^{\prime}(c)+g(c) f^{\prime}(c)$.

Theorem 2.4. (Critical point theorem). [3] If $f$ is fuzzy differentiable at a point $\boldsymbol{c}$ and $\boldsymbol{f}(\boldsymbol{c})$ is extreme value, then $\boldsymbol{c}$ is a critical point (i.e., $\boldsymbol{f}^{\prime}(\boldsymbol{c})=\mathbf{0}$ ).

Theorem 2.5. (Rolle's theorem). Let $\boldsymbol{f}$ be fuzzy continuous on $[\boldsymbol{a}, \boldsymbol{b}]$ and fuzzy differentiable on $(\boldsymbol{a}, \boldsymbol{b})$. If $\boldsymbol{f}(\boldsymbol{a})=\boldsymbol{f}(\boldsymbol{b})$, then there is one interior point $\boldsymbol{c}$ at which $\boldsymbol{f}^{\prime}(\boldsymbol{c})=\mathbf{0}$.

Proof. We assume that for all $\boldsymbol{c} \in(\boldsymbol{a}, \boldsymbol{b}), \boldsymbol{f}^{\prime}(\boldsymbol{c}) \neq \mathbf{0}$, since $\boldsymbol{f}$ is fuzzy continuous on a compact set $[\boldsymbol{a}, \boldsymbol{b}]$, it attains its maximum $\boldsymbol{M}$ and its minimum $\boldsymbol{m}$ somewhere in $[\boldsymbol{a}, \boldsymbol{b}]$. Neither extreme value is attained at an interior point (otherwise $f^{\prime}$ would vanish there) so both are attained at the end points. Since $f(\boldsymbol{a})=\boldsymbol{f}(\boldsymbol{b})$, then $\boldsymbol{M}=\boldsymbol{m}$, and hence $\boldsymbol{f}$ is constant on $[\boldsymbol{a}, \boldsymbol{b}]$. This contradicts the assumption that $\boldsymbol{f}^{\prime}$ is never $\mathbf{0}$ on $(\boldsymbol{a}, \boldsymbol{b})$. There for $\boldsymbol{f}^{\prime}(\boldsymbol{c})=\mathbf{0}$ for some $\boldsymbol{c}$ in $(\boldsymbol{a}, \boldsymbol{b})$.

Theorem 2.6. (Generalized Mean - Value Theorem). Let $\boldsymbol{f}$ and $\boldsymbol{g}$ are fuzzy continuous functions on $[\boldsymbol{a}, \boldsymbol{b}]$, and fuzzy differentiable on $(\boldsymbol{a}, \boldsymbol{b})$, assume also that there is no interior point $\boldsymbol{x}$ at which both $\boldsymbol{f}^{\prime}(\boldsymbol{x})$ and $\boldsymbol{g}^{\prime}(\boldsymbol{x})$ are infinite. Then for some interior point $\boldsymbol{c}$ we have $f^{\prime}(c)[g(b)-g(a)]=g^{\prime}(c)[f(b)-f(a)]$.

Proof. Let $\boldsymbol{h}(\boldsymbol{x})=\boldsymbol{f}(\boldsymbol{x})[\boldsymbol{g}(\boldsymbol{b})-\boldsymbol{g}(\boldsymbol{a})]-\boldsymbol{g}(\boldsymbol{x})[\boldsymbol{f}(\boldsymbol{b})-\boldsymbol{f}(\boldsymbol{a})]$. Then $\boldsymbol{h}^{\prime}(\boldsymbol{x})$ is finite if both $\boldsymbol{f}^{\prime}(\boldsymbol{x})$ and $\boldsymbol{g}^{\prime}(\boldsymbol{x})$ are finite, and $\boldsymbol{h}^{\prime}(\boldsymbol{x})$ is infinite if exactly one of $\boldsymbol{f}^{\prime}(\boldsymbol{x})$ and $\boldsymbol{g}^{\prime}(\boldsymbol{x})$ are infinite. (The hypothesis excludes the case of both $\boldsymbol{f}^{\prime}(\boldsymbol{x})$ and $\boldsymbol{g}^{\prime}(\boldsymbol{x})$ being infinite ). Also, $\boldsymbol{h}$ is fuzzy continuous on $[\boldsymbol{a}, \boldsymbol{b}]$ and $\boldsymbol{h}(\boldsymbol{a})=\boldsymbol{h}(\boldsymbol{b})=\boldsymbol{f}(\boldsymbol{a}) \boldsymbol{g}(\boldsymbol{b})-\boldsymbol{g}(\boldsymbol{a}) \boldsymbol{f}(\boldsymbol{b})$. By Rolle's Theorem we have $\boldsymbol{h}^{\prime}(\boldsymbol{c})=\mathbf{0}$ for some interior point and this proves the assertion.

Corollary 2.7. (Mean - Value Theorem ). If $\boldsymbol{f}$ is fuzzy continuous on $[\boldsymbol{a}, \boldsymbol{b}]$ and fuzzy differentiable on $(\boldsymbol{a}, \boldsymbol{b})$, then there exists $c \in(a, b)$ such that $f(b)-f(a)=f^{\prime}(c)(b-a)$.

\section{References}

[1] T. M. Apostol , Mathematical Analysis, Addison-Wesley Pub. Com., Inc., (1964)

[2] R. M. Dudley, Real Analysis and Probability, Cambridge Univ. press,(2004).

[3] S. Fuhua, The Basic Properties of Fuzzy Derivative, BUSEFAL, 76(1998),120-123.

[4] W. Rudin, Principles of Mathematical Analysis, McGraw-Hill, Inc., (1976).

[5] A. K. Katsaras, Fuzzy Topological Vector Spaces I, Fuzzy Sets and Systems, 6 (1981) 85-95 